\title{
Entre l'art de l'invisibilité et l'art de la démesure : Marcel Proust caricaturé par Jean Cocteau
}

Nous savons aujourd'hui quel'un des plus grands soucis de Marcel Proust, dès la mise en marche du projet d'écrire $A$ la recherche $d u$ temps perdu, a été le sort de son œuvre et que, enfermé dans sa chambre de liège, il s'adonnait, en plus de la rédaction des volumes successifs de la Recherche, à l'élaboration de stratagèmes ayant pour but la préparation d'un accueil favorable de son roman par la critique ${ }^{1}$.

L'attribution du prix Goncourt, le 10 décembre 1919 à À l'ombre des jeunes filles en fleurs, a éveillé une véritable querelle médiatique et littéraire tournant plus autour de la personne de l'écrivain que de son ouvre 2 . Proust, court vainqueur par deux voix face à son concurrent direct Roland Dorgelès, l'auteur du roman Les croix de bois, a paru, aux yeux de certains journalistes, trop âgé pour devenir lauréat d'un prix en principe destiné à récompenser un jeune écrivain. C’est pour cela que, comme le dévoile Thierry Laget (2019), l'auteur a entrepris, entre autres, de divulguer des copies photographiques de son fameux portrait de jeunesse peint par Jaques Émile Blanche. Dans ce contexte-là, le dessin de Jean Cocteau, daté de 1920, représentant un Marcel Proust vieux et fatigué dans son célèbre lourd manteau, possède sans doute tous les traits d'une image autant provocatrice que caricaturale ${ }^{3}$. Effet d'une rivalité avec Proust, sinon manière de déjouer des stratégies autofictionnelles de celui-ci

\footnotetext{
Katarzyna Thiel-Jańczuk - maître de conférences à l'Institut d'Études Culturelles de l'Université Adam Mickiewicz à Poznań. Adresse de correspondance : l'Institut d'Études Culturelles, Université Adam Mickiewicz, ul. Szamarzewskiego 89 A, 60-568 Poznań, Pologne ; e-mail : katka@o365.amu.edu.pl

ORCID iD : https://orcid.org/0000-0002-9418-5231

1. Michel Schneider, par exemple, dévoile les stratagèmes de Proust dans son essai biographique consacré à Proust : (2014). L'auteur, l'autre. Proust et son double. Paris : Gallimard.

2. Cette querelle fait l'objet d'un essai de Thierry Laget, Proust, prix Goncourt : une émeute littéraire (2019).

3. Le dessin de Cocteau est accessible sur le site internet suivant : https://www.centrepompidou.fr/ en/ressources/oeuvre/ckAzRLB
} 
aussi bien sur le plan de l'écriture que sur celui de la «visibilité »", ce dessin, loin de traduire un simple désir de moquerie, me semble emblématique d'une tentative de démythifier la position de Proust dans l'histoire littéraire, à l'image de ce que propose Claude Arnaud dans son essai Proust contre Cocteau (2013) 5 . Dans mon article, je voudrais analyser, à partir de l'essai évoqué, l'apport du dessin de Cocteau à l'abolition d'un mythe que la critique universitaire des années 1950 a instauré autour de Marcel Proust, ainsi que réfléchir plus généralement sur les relations entre la caricature visuelle et l'art du pastiche, "cette sorte de caricature phonétique et verbale " (Keller, 2016, p. 167) pratiquée par Proust lui-même.

\section{Proust contre Cocteau ou l'art de l'invisibilité}

Proust et Cocteau font connaissance vers 1910 par l'intermédiaire de Lucien Daudet, ami intime de Marcel, au moment où celui-ci, retiré depuis quelques années dans son appartement boulevard Haussmann après la mort de ses parents, entreprend la rédaction du premier volume de la Recherche qui a évolué d'un essai critique entamé deux ans plus tôt et connu sous le titre Contre Sainte-Beuve. Leurs biographies se ressemblent sur plusieurs points : origine bourgeoise, attachement à la mère, formation au lycée Condorcet, orientation homosexuelle, fréquentation de salons, goût pour la moquerie et le pastiche. La différence d'âge (vingt-et-un ans pour Cocteau contre près de quarante ans pour Proust) explique, en partie seulement, la complexité de leur relation déterminée avant tout, comme le suggère Claude Arnaud, par des façons différentes d'aborder la question de la célébrité. Or celle-ci, à l'approche de l'âge de la "reproductibilité technique " (Walter Benjamin) qui a rendu possible la diffusion des images à grande échelle et le développement de médias visuels, n’a plus la même valeur qu'à la Belle Époque des salons mondains dont il est question dans la Recherche. Nathalie Heinich affirme : «Avec l'élite médiatique du XX ${ }^{\mathrm{e}}$ siècle, la visibilité a remplacé la mondanité » (2013, p. 59). Si donc Proust, qui s'oppose dans Contre Sainte-Beuve à une idée de la littérature entendue comme l'art de la conversation mondaine $e^{6}$, favorise «l'œuvre » comme source de gloire, Cocteau situe celle-ci du côté de «l'homme $»^{7}$ :

4. Par « visibilité » Nathalie Heinich comprend la production d'un « effet de présence » d'une personne dans la société, grâce avant tout à la « reproduction technique des visages à grande échelle » (2013, p. 17 et 32 ).

5. Le dessin de Proust par Cocteau se trouve en couverture de l'ouvrage d'Arnaud, à côté du portrait de 1921 de Cocteau par Francis Picabia.

6. Rappelons la fameuse phrase proustienne : « un livre est le produit d'un autre moi que celui que nous manifestons dans nos habitudes, dans la société, dans nos vices» (Proust, 1954).

7. Selon José-Luis Diaz (2011), cette dialectique entre "l'homme " et "l'œuvre », constitutive des approches biographique ou anti-biographique des œuvres littéraires, désigne des étapes de l'histoire de critique littéraire depuis l'âge classique. 
il recherchait avidement - constate Claude Arnaud - cette forme démultipliée d'amour, en véritable diabétique de la gloire : seule la présence d'une foule enthousiaste pouvait lui assurer ce sentiment de plénitude que même le voisinage radioactif de sa mère ne pouvait plus lui offrir. (2013)

Cocteau ne croit bien évidemment pas au retour à la mondanité de la Belle Époque ni à ses salons et il est conscient que, peu à peu, une nouvelle élite émerge dont la position sociale ne dépend plus de qualités morales ou de capital financier, mais de l'effet de présence qu'elle est capable de créer dans la société. Or, si l'auteur du Potomak ne cache pas sa fascination pour la gloire qui l'intéresse tant pour lui-même que chez les autres, il la comprend, conformément à l'esprit de son époque, avant tout en termes de visibilité : l'expression "monstres sacrés " qu'il utilise pour appeler des personnalités lui « inspir[ant] des sentiments quasi religieux » (Milorad, 1979, p. 10) se réfère en fait à l'image de ces personnalités qui circulent dans la société, qu'il s'agisse des hommes de lettres des époques passées, tels Paul Verlaine ou Arthur Rimbaud, ou des représentants contemporains de l'univers du "spectacle ", telle Brigitte Bardot. Dans Essai de critique indirecte, à côté de la réflexion sur le problème de « la personnalité de grand format » qui l'intrigue, Cocteau fait, par exemple, cette remarque à propos de la fameuse barbe de Victor Hugo :

Je pense aux photographies révélatrices de l'exil. On ignorait encore les dangers de la chambre noire. Hugo sans barbe ! Il l'a vite remise, le malin. Settembrini riposte : "Les comédiens ». Mais les comédiens possèdent aussi leurs grands formats. (Cocteau, 1932)

Devenir visible apparaît donc comme l'enjeu de la société moderne et Cocteau s'y emploie avec application, il semble même être en avance par rapport à son époque : à l'instar des écrivains d'aujourd'hui qui, comme le constate Vincent Kaufmann, lorsqu'ils veulent être lus doivent « être aussi capable[s] de se faire voir » (2017), l'auteur des Enfants terribles réussit à imposer à ses contemporains aussi bien son œuvre que sa personne. À côté de genres littéraires traditionnels, il a volontiers recours aux arts visuels $^{8}$, se faisant connaître aussi bien comme poète, écrivain et dramaturge que dessinateur, peintre, photographe ou cinéaste, voire même acteur (il joue, par exemple, le rôle du baron Julius Carol dans Le Baron fantôme de Serge de Poligny de 1942 ou son propre rôle de poète dans Le Testament d'Orphée qu'il tourne en 1960). Il n'hésite pas non plus à exhiber certains aspects de sa vie intime, notamment ceux qui concernent

8. Soulignons également que, suivant les mouvements avant-gardistes de l'époque, Cocteau entame un dialogue incessant avec les beaux-arts, allant jusqu'à l'attention portée à la dimension graphique de ses poèmes. David Gullentops propose le terme de " lisuel » pour décrire ce mariage du textuel et du visuel caractéristique de la poésie de Cocteau (cité dans Zawadzka, 2014, p. 81-85). 
ses relations homosexuelles 9 . Il s'autoportraiture à plusieurs reprises et est également portraituré par les plus importants artistes de son époque (pour ne mentionner que Christian Bérard, Jacques-Émile Blanche, Moïse Kisling, Dora Maar, Amadeo Modigliani, Pablo Picasso, et plusieurs autres). Beaucoup plus polyvalent que Proust, dont la principale activité créatrice se limite à l'écriture ${ }^{10}$, il semble aussi beaucoup plus efficace que l'auteur de la Recherche dans la création de son image publique.

Contrairement à Cocteau, qui sait habilement joindre un "moi mondain » et un " moi profond ", la fréquentation des salons, en dehors de procurer de la matière pour son roman, n’aura pas pour Proust de conséquences positives : sa réputation de "snob " et de " chroniqueur mondain du Figaro " fera qu'André Gide refusera la publication de $D u$ côté de chez Swann chez la maison d'édition Gallimard ${ }^{11}$. De même, sa modeste production littéraire avant l'édition de Swann en 1913 (qu'il publie, comme on le sait, à compte d'auteur) - Les Plaisirs et les jours (1896), des traductions de Ruskin et quelques articles - fait de lui un écrivain pratiquement inconnu de la critique au moment du prix Goncourt ce qui rend, pour la presse de l'époque, le choix du jury presque incompréhensible. Déjà Cocteau, au moment de leur première rencontre, est conscient des différents talents de Proust mais refuse de le considérer comme un maître, celui-ci n'ayant pas une autorité littéraire confirmée par une publication littéraire étoffée, et c'est plutôt l'auteur du Prince frivole qui jouera auprès de son collègue aîné un rôle de mentor et de promoteur ${ }^{12}$. Arnaud affirme : "Si Cocteau ne peut s'identifier à Proust, c'est d'abord que Proust n'existe pas, stricto sensu : il est avant tout ce que sa pensée vagabonde et ses projections changeantes le font devenir » (2013). En effet, malgré le prix Goncourt, Proust ne s'impose pas sur la scène littéraire française et sera longtemps considéré, presque à l'unanimité, comme un écrivain "malade, ennuyeux, interminable ", que l'on situe, faute de mieux, parmi les héritiers de la tradition classique (Tadié, 1983, p. 183) ${ }^{13}$. En 1949, il disparaît littéralement du Panorama de la nouvelle littérature française préparé par Gaëtan Picon qui, se sentant obligé d'expliquer aux lecteurs cet acte d'exclusion tout à fait conscient, affirme :

9. Rappelons que Cocteau est l'un des premiers écrivains, à côté d'André Gide et Jean Genet, à avoir contribué au dévoilement homosexuel en France, avec notamment Le livre blanc de 1928.

10. Cela ne veut pas dire que Proust n'a pas pratiqué d'autres arts, mais sans grand succès : on connaît ses dessins faits le plus souvent à la marge des cahiers et carnets manuscrits de la Recherche ou des lettres à ses amis. Philippe Sollers leur a même consacré un essai intitulé L'oeil de Proust. Les dessins de Marcel Proust (1999). Yves Sandre évoque également les pastiches d'œuvres musicales réalisées par Proust dont il parle dans ses lettres (Sandre, 1971, p. 688).

11. La citation vient d'une lettre d'excuses, envoyée par Gide à Proust, en janvier 1914 (Dupuis, 2013).

12. Cocteau est l'un des premiers à saluer la parution de Du côté de chez Swann à la demande de Proust lui-même dans Excelsior du 23 novembre 1913.

13. Pour plus de détails, concernant cette phase précoce de la réception de l'œuvre proustienne, je renvoie à la troisième partie de la publication de Jean-Yves Tadié intitulée «Bilan critique ». 
Si je ne parle pas de Proust, ce n'est pas parce que je l'ignore ou que je le conteste : c'est que son œuvre s'est éloignée de nous non seulement par sa date, mais par sa nature parce qu'elle est le couronnement génial d'un symbolisme et d'un individualisme psychologique et analytique momentanément sans action sur nous. (cité dans Tadié, 1983, p. 187)

Dans ce contexte, l'enfermement de l'auteur de la Recherche dans sa chambre de liège, loin dêtre considéré par ses contemporains comme un geste sacrificiel de soumission de la vie à l'œuvre, contribue plutôt à renforcer l'image négative de Proust dans la société que Cocteau d'ailleurs, le comparant souvent au capitaine Nemo ou à la séquestrée de Poitiers, contribue à maintenir (Sakamoto, 2013, p. 32). Comme le dévoile Jean-Yves Tadié, il faut attendre les années 1950 pour que Proust soit sorti de l'oubli grâce notamment à la publication d'À la recherche de Marcel Proust d'André Maurois (1949), ainsi que de l'ensemble de son cycle dans la collection de la Pléiade, et de deux inédits, Jean Santeuil (1952) et Contre Sainte-Beuve (1954). Ces deux dernières publications auront non seulement une influence décisive sur le renouveau de la critique littéraire en France, mais contribueront à changer la perception que l'on se faisait du légendaire isolement proustien. Bernard de Faillois, dans l'introduction à son édition de Contre Sainte-Beuve, explique :

La simple vue des manuscrits prouve [...] que [Proust] n’a jamais cessé de travailler, que jamais peut-être une création ne fut à ce point continue. Mais cette continuité ne pouvait apparaître, parce que certaines périodes étaient restées dans l'ombre. Entre 1896 où paraissent Les Plaisirs et les Jours, et 1910 où il commence à rédiger la Recherche, on ne trouvait que deux traductions de Ruskin et quelques articles. C'était peu. Cela suffisait en effet pour imaginer une existence oisive, consacrée à l'art, au monde, à l'amour, et totalement absorbée par eux. Mais on ignorait alors les travaux les plus importants de ces quinze années [...] Jean Santeuil et [...] Contre Sainte-Beuve. (1954, p. 12-13)

Cependant, la Nouvelle Critique, si elle a abolit un mythe proustien, en a créé un autre. Reconnu, à la suite du philologue autrichien Léo Spizer, comme un styliste entièrement impliqué dans son œuvre et n'ayant d'existence en dehors d'elle, l'auteur de Jean Santeuil, considéré comme un fou invisible, devient désormais le héros d'une littérature autarcique, voire un «Saint-Proust ${ }^{14}$ qui n'a plus besoin de se soucier de sa visibilité $^{15}$. Il est significatif que, dans ce nouveau contexte, marqué, d'un côté, par l'anti-biographisme structuraliste et poststructuraliste et, de l'autre, par l'émergence d'une réflexion critique à propos de l'influence des médias sur la société, c'est la réception de Cocteau qui change radicalement, faisant de fait basculer l'écrivain dans

14. Allusion à l'ouvrage de D. Maingueneau, Contre Saint Proust ou la fin de la Littérature (2006).

15. Je signale déjà cette déconstruction du mythe proustien par la Nouvelle Critique dans mon article Proust invisible? Quelques remarques autour de Proust, Prix Goncourt. Une émeute littéraire de Thierry Laget (à paraître). 
l'oubli. Un demi-siècle après sa mort, Philippe Sollers résume le jugement critique que la postérité a réservé à l'auteur du Prince frivole : "Cocteau il y a cinquante ans était encore un virtuose de la Société du Spectacle (Debord le hait pour cette raison) » $(2012)^{16}$. Mais déjà Cocteau lui-même, vers la fin de sa vie, semble avoir découvert des inconvénients à la visibilité :

Il y a un isolement étrange et paradoxal de Cocteau - constate Claude Arnaud Il connaît tout le monde, il est passé d'une réputation d'avant-garde à l'Académie française, il est poète, écrivain, dramaturge, peintre, cinéaste, il sort, il brille, il travaille, ses journées sont remplies à ras bord, il est persuadé d’avoir du génie, mais il n'arrête pas de s’étonner qu'on lui refuse ce titre. «Je suis un fantôme sans château », dit-il dans son Journal des années 1960-1961 (il meurt en 1963). Autrement dit : je suis très visible et pourtant invisible, on me dévisage, mais on ne «m’envisage » pas, un sort maléfique me poursuit, « on a toujours parlé de moi avec une scrupuleuse inexactitude ». (2013)

À « l'âge d[u] Style " (Maingueneau), c'est l'invisibilité qui devient la marque $\mathrm{du}$ " grand-écrivain $»^{17}$.

\section{Cocteau contre Proust ou l'art de la démesure}

Quelle est donc, face aux remarques qui précèdent, l'utilité du dessin de Cocteau ? Que nous dit-il sur les relations entre les deux écrivains? Quel est son rôle dans la construction ou, au contraire, la déconstruction du mythe proustien ? Comment pouvons-nous le comprendre aujourd'hui, dans le nouveau contexte théorique et médiatique ? Dans La Difficulté dêtre, publié en 1947, donc près de quarante ans après leur première rencontre et vingt-cinq ans après la mort de Proust, nous trouvons un fragment qui crée sans doute une relation ekphrastique avec le dessin en question :

[...] [I]l rentrait à l'aube en croisant sa pelisse, blême, les yeux cernés de bistre, un litre d'eau d'Évian dépassant de sa poche, sa frange noire sur le front, une de ses bottines à boutons déboutonnée, son chapeau melon à la main, pareil au spectre de Sacher Masoch. (Cocteau, 1964, p. 69-70)

16. Le concept de «société du spectacle » a été forgé par le philosophe Guy Debord en 1967 et renvoie à une critique de la société de consommation où « le rapport social entre des personnes est médiatisé par des images » (1992, p. 10).

17. Nathalie Heinich affirme une relation paradoxale entre la littérature et les médias lorsqu'elle dit : «Le discrédit attaché à la visibilité dans le monde littéraire est si fort que peuvent même s’y produire des stratégies d'invisibilité, d'ailleurs relativement rentables en matière de reconnaissance [...]" (2013, p. 161). 
Le dessin comme le portrait écrit se réfèrent à la période où Proust, vieux et malade, sortait la nuit pour chercher dans des endroits mondains, tel le Ritz, des modèles de personnages pour son roman. Ce qui frappe dans les deux représentations, c'est l'évocation du manteau dont Proust, toujours soucieux de sa santé et ayant peur du moindre courant d'air, avait l'habitude de s'envelopper, même à table. Ce manteau fait également penser à celui que Proust, tentant de séduire Cocteau, a voulu insidieusement lui offrir : un geste surprotecteur qui n’a pas seulement mis fin à leur amitié, mais qui a permis à Cocteau de se rendre compte du caractère manipulateur de l'auteur de la Recherche. Arnaud rappelle dans son essai que les amis de Marcel sont souvent partagés entre leur admiration pour son génie et la réprobation de la «tyrannie affective " qu'il leur impose, une espèce "d'horrible manie qu'a Proust d'enfermer les êtres pour les avoir à soi » (2013). Le Marcel réel, sur plusieurs plans de sa vie familiale et sociale, est une personne démesurée, voire même caricaturale :

Il est trop. Trop amoureux de cette génitrice, qui prône pourtant la logique et le raisonnable. Trop sensible aux parfums de fleur ou de femme, comme aux variations de la lumière. Trop attaché aux meubles qui forment le décor apaisant de la maison d'Auteuil où il naquit. Trop généreux avec les domestiques et les clochards, qu'il comble de billets, comme s'il cherchait là encore une reconnaissance disproportionnée. Trop modeste, suppliant et compassionnel aussi. (Arnaud, 2013)

Ainsi, le caractère de l'écrivain est non seulement compatible avec les reproches que la critique adressait à son œuvre (" trop long, trop difficile, trop saturé de références artistiques et privé d'action pour être commercialisable " (Arnaud, 2013)), mais aussi, en général, avec son goût pour l'imitation. Yves Sandre, dans son commentaire à l'édition des Pastiches dans la Pléiade affirme :

Nous avons plus d'une preuve que la personnalité profonde de Proust est intervenue à tous les niveaux dans la rédaction de ses pastiches. Ils s'expliquent d'abord par le tempérament et les dispositions particulières de l'écrivain. Caractérologiquement [...] d'une sensibilité exceptionnelle, encore renforcée par une polarité vénusienne très nette, qui apparait dans une extrême perméabilité à toutes les influences. Ne disons pas que Proust s'adonne aux pastiches parce qu'il est homosexuel, mais disons que son inversion et sa tendance au pastiche sont les symptômes concordants d'un état de « déséquilibre » psychosomatique. (1971, p. 690)

Si donc pour Sandre, en 1971, la relation entre l'état de déséquilibre éprouvé par Proust et ses pastiches est incontestable (Arnaud semble d'ailleurs partager cette opinion lorsqu'il parle d'une "cruauté teintée de dérision, propre à la culture homosexuelle d'alors » (2013) qui devait caractériser l'auteur des Pastiches), il les envisage, conformément à la vulgate structuraliste, avant tout comme des exercices de style qui ont préparé « les voies du roman à venir », donc selon une optique strictement 
littéraire. Or, s'il faut admettre que Proust, au moins à partir du moment où il se met à écrire son œuvre majeure, attribue lui-même, après coup, une finalité littéraire à ces exercices, il est difficile d'oublier que les jeux d'imitation faisaient également partie de sa façon d'être dans le monde et qu'ils avaient parfois des conséquences négatives pour l'image du jeune Marcel dans la société. Luzius Keller (2016), qui insiste sur l'existence d'une relation stricte entre les pastiches littéraires de Proust et ces imitations mondaines, évoque ainsi un conflit qui s'est produit entre Robert de Montesquiou, Marcel Proust et son ami Lucien, lors d'un dîner chez la famille Daudet en 1895, quand les jeunes gens se sont permis de parodier la mimique, la manière de parler et les gestes du fameux dandy. Dans le recueil des textes satiriques Délices de Capharnaüm, Montesquiou évoque cet épisode en faisant à l'occasion une critique des imitations pratiquées par les deux amis et il situe celles-là bien au-dessous de l'art de la caricature qui florissait à l'époque de fin de siècle :

[C]eux qui s'essaient à réaliser ce qu'ils croient être des imitations, ce qu'ils désignent ainsi ; je ne parle point de cette imitation noble, laquelle s'applique à prendre pour exemples, de vrais mérites, mais cette pauvre simulation simiesque, appliquée à reproduire des intonations, à singer des attitudes; sorte de caricature phonétique et verbale, soulignée par la grimace et le geste, avec cette infériorité morale sur les albums publiés par nos humoristes, qu'elle fait succéder aux protestations de déférence écrite et parlée, envers les modèles, l'essai de les ravaler, en leur absence, à l'emploi de divertissement d'après-dîner, pour un public friand de ces simagrées. (cité dans Keller, 2016, p.167)

Nous voyons donc que Montesquiou ne condamne pas les pratiques imitatrices en tant que telles, mais leur dissimulation par deux jeunes gens et c'est en fait elle qui, une quinzaine d'années plus tard, rendra suspectes à Cocteau les déclarations proustiennes de séparation de la vie et de l'œuvre, cruciales tant pour la forme définitive de son roman que pour le statut particulier de son narrateur. Comme si l'auteur des Enfants terribles, ne voulant pas être dupe, n'y voyait qu'une nouvelle manifestation du caractère démesuré d'un Proust prenant la pose d'un "fou guetté par le génie " et " dissimulant cette prétention sous des excès de modestie " (Arnaud, 2013), et qui laissera Cocteau indifférent à son futur culte. Comme le remarque très justement Arnaud, Cocteau n'a pas lu Contre Sainte-Beuve :

Quiconque aborde aujourd'hui la Recherche ne lit évidemment pas le même livre que Cocteau. Il est fort du Contre Sainte-Beuve qu'un éditeur publia en 1954, en rassemblant arbitrairement des textes épars de Proust, et qui aida Nouvelle Critique à décréter «la mort de l'auteur ». (2013)

Faudrait-il donc que nous, lecteurs venus après Contre Sainte-Beuve, considérions " la mort de l'auteur " comme une forme, ultime et immodérée, de camouflage ? Les paroles suivantes de l'auteur de la Recherche ne sont-elles que la dissimulation 
d'un nouveau jeu où Marcel Proust veut seulement faire semblant de devenir invisible?

Le tout était surtout pour moi affaire d'hygiène, il faut se purger du vice naturel d'idolâtrie et d'imitation. Et au lieu de faire sournoisement du Michelet ou du Goncourt en signant (ici les noms de tels ou tels de nos contemporains les plus aimables), d'en faire ouvertement sous forme de pastiches, pour redescendre à ne plus être que Marcel Proust quand j’écris mes romans. (cité dans Sandre, 1971, p. 690)

Si Proust a sans doute connu les plus grands caricaturistes de son époque et les a inspirés ${ }^{18}$, il paraît étonnant, vu son caractère outré et la réputation d'imitateur qui courait sur lui, qu'il n'existe apparemment pas, à part le dessin de Cocteau, de portraits-charges contre lui conçus de son vivant ${ }^{19}$. Déjà celui-là, tout en comportant quelques aspects comiques, propres à la représentation caricaturale d'une personne (déformation de la main et des pieds, regard pitoyable, énorme manteau, cheveux non coupés, chapeau abîmé), oscille en fait entre une caricature et un dessin, conformément à l'idée de Cocteau selon laquelle les frontières entre les deux arts sont floues, une caricature tirant sa valeur humoristique d'un « air épinglé vif » d'un personnage plutôt que d'une représentation transformant certains traits de son modèle (1959, p. 3). Contrairement donc à Proust, qui attribue une déformation caricaturale des personnes à l'action destructrice du temps ${ }^{20}$, Cocteau paraît l'attacher plutôt à une fonction démythifiante de son dessin, à la relation que celui-ci établit avec l'image de l'auteur de la Recherche circulant dans la société. Même si nous ne pouvons pas savoir aujourd'hui si Proust a vu le dessin, qui probablement faisait partie de l'archive privée de l'auteur de Thomas l'imposteur ${ }^{21}$, nous pouvons parier que Cocteau, en revanche, a dû voir l'édition du volume des Jeunes filles en fleurs, accompagnée de la copie du portrait de son auteur par Blanche et qu'il connaissait les reproches, déjà mentionnés, que la presse adressait à Proust après le prix Goncourt. En présentant sur le dessin un homme tout à fait différent de celui qui était donné à voir

18. Il a sans doute rencontré le plus grand caricaturiste de la Belle Époque, le célèbre Sem (Georges Goursat), qui fréquentait le salon de la grande amie et protectrice de Proust, Madeleine Lemaire. Celuilà n’a pourtant pas immortalisé l'écrivain. En revanche, plusieurs personnes qu'il a dessinées ont servi de modèles aux personnages proustiens ; voir Uro., Y. (2005). Les amis et modèles de Proust dans l'ouvre de Sem.

19. Les caricaturistes tels que Ferdinand Bac ou Leonetto Cappiello ont portraituré Proust, mais sans véritablement appliquer des modifications typiques de ce genre de représentations.

20. C'est le grand thème de la dernière partie du Temps retrouvé, consacrée au vieillissement, aux changements des corps et à la déformation des traits du visage.

21. Le dessin fait actuellement partie du fond Dermit et a été présenté dans la maison de Jean Cocteau à Milly-la-Forêt dans le cadre de l'exposition Jean Cocteau. Dessins d'une vie. Du Centre Pompidou à Milly-la-Forêt, du $1^{\text {er }}$ juin à 27 octobre 2019. Je n’ai pas réussi à vérifier s'il a été exposé du vivant de Proust. 
à un large public, et à la fois l'homme, semble-t-il, beaucoup plus réel qu'il connaissait et qu'il ne réussissait pas à oublier, il a sans doute voulu ridiculiser son ancien ami et rival et son dévouement exagéré, voire même martyrologique, à son œuvre. À l'âge de style, qui vénérait un narrateur proustien universel, le dessin rappelant trop « le petit Marcel » particulier, pouvait être considéré comme la preuve d'une absence de perspicacité de son créateur ou, bien au contraire, une contribution involontaire à sa future renommée. Aujourd'hui, à une époque que l'on pourrait appeler " postsainte-beuviste ", il pourra être apprécié en tant que contribution au dévoilement d'une - pour parler en termes proposés par Jérôme $\mathrm{Meizoz}^{22}$ - "posture » de l'auteur de la Recherche, qui complète et renforce sur le plan visuel, longtemps négligé par le discours biographique traditionnel, son " ethos discursif ", voire des stratégies d'invisibilité et de démesure mises en œuvre dans son roman.

Mais, que Cocteau le veuille ou non, la meilleure illustration de ce jeu entre l'universel et le particulier, dans lequel est mêlé son dessin, est apportée par le narrateur proustien dans le Temps retrouvé découvrant, derrière les visages de ses amis vieillis par le temps, la véritable puissance de la caricature :

[...] en admirant le vieil ermite aux cheveux blancs quétait devenu Legrandin, tout d'un coup je constatai [...] dans le méplat de ses joues, la construction de celles de son jeune neveu, Léonor de Cambremer, qui pourtant avait l'air de ne lui ressembler nullement ; à ce premier trait commun j'ajoutai un autre que je n'avais pas remarqué chez Léonor de Cambremer, puis d'autres et qui n'étaient aucun de ceux que m’offrait d'habitude la synthèse de sa jeunesse, de sorte que j'eus bientôt de lui comme une caricature plus vraie, plus profonde, que si elle avait été littéralement ressemblante ; son oncle me semblait maintenant seulement le jeune Cambremer ayant pris pour s'amuser les apparences du vieillard qu'en réalité il serait un jour, si bien que ce neetait plus seulement ce quétaient devenus les jeunes d'autrefois, mais ce que deviendront ceux d'aujourd'hui, qui me donnait avec tant de force la sensation du temps. (Proust, 1999, p. 2320-2321)

\section{RÉFÉRENCES}

Arnaud, C. (2013). Proust contre Cocteau. Paris : Grasset (ebook, sans foliotage).

Cocteau, J. (1932). Essai de critique indirecte. Paris : Grasset (ebook, sans foliotage).

Cocteau, J. (1959). Éloge de la caricature. Dans Plus on est de fous... : les meilleurs dessins d'Europe (p. 3-4). Paris : Hachette.

Cocteau, J. (1964). La Difficulté d'être. Paris : Union Générale d'Éditions.

22. Je fais ici l'allusion à l'œuvre de Jérôme Meizoz. (2016). La littérature "en personne ». Scène médiatique et formes d'incarnation. Genève : Slatkine Érudition. 
Diaz, J.-L. (2011). L'homme et l'œuvre. Contribution à une histoire de la critique. Paris : Presses Universitaires de France.

De Faillois, B. (1954). Préface. Dans M. Proust. Contre Sainte-Beuve (p. 9-50). Paris : Gallimard. Debord, G. (1992). La société du spectacle. Paris : Gallimard.

Dupuis, J. (2013). "Je m'accuse » de Gide à Proust aux enchères. L'Express. https://www. lexpress.fr/culture/livre/le-je-m-accuse-de-gide-a-proust-aux-encheres-le-26-novembre_1293373.html

Heinich, N. (2013). De la visibilité. Excellence et singularité en régime médiatique. Paris : Gallimard.

Kaufmann, V. (2017). Dernières nouvelles du spectacle (ce que les médias font à la littérature). Paris : Seuil (ebook, sans foliotage).

Keller, L. (2016). Autoportrait, portrait et caricature dans la période fin de siècle. La comtesse de Castiglione, Robert de Montesquiou, Marcel Proust. Dans L. Keller, Lire, traduire, éditer Proust (p. 155-170). Paris : Classiques Garnier.

Laget, T. (2019). Proust, prix Goncourt : une émeute littéraire. Paris : Gallimard (ebook).

Maingueneau, D. (2006). Contre Saint Proust ou la fin de la Littérature. Paris : Belin.

Milorad. (1979). Introduction. Dans J. Cocteau, Mes monstres sacrés (p. 9-14). E. Dermit et B. Meyer (éd.). Paris : Encre.

Proust, M. (1954). Contre Sainte-Beuve. Paris : Gallimard.

Proust, M. (1999). À la recherche du temps perdu. Le Temps retrouvé. Paris : Gallimard.

Sakamoto, H. (2013). Jean Cocteau. Dans J.-Y. Tadié (dir.), Le Cercle de Marcel Proust (p. 27-36). Paris : Honoré Champion.

Sandre, Y. (1971). Pastiches et mélanges. Note sur le texte. Dans M. Proust. Contre SainteBeuve précédé de Pastiches et mélanges et suivi de Essais et articles (p. 687-694). Paris : Gallimard.

Sollers, Ph. (1999). L'œil de Proust. Les dessins de Marcel Proust. Paris : Stock.

Sollers, Ph. (le 6 décembre 2012). Cocteau, le sociétaire du Spectacle. Le Nouvel Observateur. http://www.pilefacebis.com/sollers/IMG/pdf/Cocteau $\% 201$ e $\% 20$ socie $\%$ CC $\% 81$ taire $\% 20$ du\%20Spectacle.pdf

Tadié, J.-Y. (1983). Proust, le dossier. Paris : Belfond.

Uro, Y. (2005). Les amis et modèles de Proust dans l'œuvre de Sem. Feuillets Sem, 53, 3-13.

Zawadzka, M. (2014). Jean Cocteau : le poète face aux arts plastiques. Warszawa : Wydawnictwa Uniwersytetu Warszawskiego.

RÉSUMÉ : Partant d'un dessin de Marcel Proust par Jean Cocteau conçu en 1922 ainsi que d'un essai biographique de Claude Arnaud publié en 2013, l'auteur de l'article présente la relation entre les deux écrivains en référence à la problématique de leur " visibilité » (Nathalie Heinich). Dans ce contexte-là, le dessin de Cocteau, restant en accord avec le caractère démesuré de Marcel Proust, peut être aujourd'hui considéré comme une représentation caricaturale de l'auteur de la Recherche qui démythifie une image de Proust-écrivain invisible créée par la vulgate structuraliste. Aussi, dans le contexte médiatique et théorique contemporain, la représentation visuelle de Proust par Cocteau peut s'avérer utile pour la réflexion à propos de la « conduite » 
non-narrative de Proust qui, à côté de son activité discursive, définit la " posture " (Jérôme Meizoz) de l'auteur de la Recherche dans le champ littéraire français.

Mots-clés : Proust, Cocteau, visibilité, démesure, caricature

\section{Between the art of invisibility and the art of exaggeration:} Marcel Proust caricatured by Jean Cocteau

ABSTRACT: The paper discusses the relationship between Marcel Proust and Jean Cocteau in connection with the problem of their "visibility" (Nathalie Heinich) based on Jean Cocteau's portrait (1922) of Marcel Proust and Claude Arnaud's biographical essay (2013). In this context, Cocteau's drawing, which conveys Marcel Proust's extravagant character, could nowadays be considered not only a caricatural representation of the author of In Search of Lost Time, but also a demythologizing one, especially in contrast to the image of invisible Proust created by structuralist interpretations. Also, in the mediatic and theoretical contemporary context, Cocteau's visual representation of Proust could be used to reflect on Proust's action (conduite) which, combined with his narrative discourse, defines the "posture" (Jérôme Meizoz) of the author of In Search... within the literary field.

Keywords: Proust, Cocteau, visibility, exaggeration, caricature 